\title{
Stable magnetostructural coupling with tunable magnetoresponsive effects in hexagonal ferromagnets
}

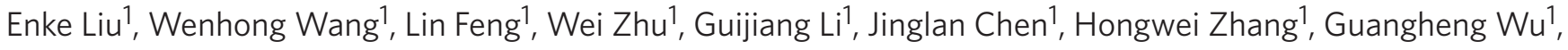
Chengbao Jiang ${ }^{2}$, Huibin $\mathrm{Xu}^{2} \&$ Frank de Boer ${ }^{3}$

The magnetostructural coupling between the structural and the magnetic transition has a crucial role in magnetoresponsive effects in a martensitic-transition system. A combination of various magnetoresponsive effects based on this coupling may facilitate the multifunctional applications of a host material. Here we demonstrate the feasibility of obtaining a stable magnetostructural coupling over a broad temperature window from 350 to $70 \mathrm{~K}$, in combination with tunable magnetoresponsive effects, in MnNiGe:Fe alloys. The alloy exhibits a magneticfield-induced martensitic transition from paramagnetic austenite to ferromagnetic martensite. The results indicate that stable magnetostructural coupling is accessible in hexagonal phasetransition systems to attain the magnetoresponsive effects with broad tunability.

\footnotetext{
1 State Key Laboratory for Magnetism, Beijing National Laboratory for Condensed Matter Physics, Institute of Physics, Chinese Academy of Sciences, Beijing 100190, China. ${ }^{2}$ School of Materials Science and Engineering, Beihang University, Beijing 100191, China. ${ }^{3}$ Van der Waals-Zeeman Instituut, Universiteit van Amsterdam, Amsterdam, Netherlands. Correspondence and requests for materials should be addressed to W.H.W. (email:wenhong.wang@iphy.ac.cn).
} 
T he ferromagnetic martensitic transition (FMMT) ${ }^{1-3}$, a coinciding crystallographic and magnetic transition mainly found in Fe-based and Heusler ferromagnetic alloys, is receiving increasing attention from both the magnetism and the material science community due to the massive variations of associated magnetoresponsive effects, such as magnetic-field-induced shape memory/strain effect ${ }^{4-7}$, magnetoresistance ${ }^{8,9}$, Hall effect ${ }^{10}$ and magnetocaloric effect ${ }^{11,12}$. These effects are of interest for many potential technological applications like magnetic actuators ${ }^{13,14}$, sensors ${ }^{15}$, energy-harvesting devices ${ }^{16}$ and solid-state magnetic refrigeration ${ }^{17}$. In these functionalities, the magnetostructural coupling between the structural and the magnetic transition has an essential role. Seeking a stable coupling in a broad temperature range is a scientific and technological challenge.

In the case of ferromagnetic phase transitions coupled with martensitic-like structural changes, it is the ferromagnetic ordering (for example, spontaneous magnetization) that triggers modest structural modifications due to the magnetoelastic coupling ${ }^{18}$. These magnetoelastic transitions have been utilized in the intensive investigations of a large body of giant magnetocaloric materials ${ }^{19-27}$. In contrast, in typical FMMTs, the change of structural symmetries of austenite and martensite is remarkable ${ }^{6-11}$. The transition converts the different magnetic states (moment values and type of coupling) in between the two phases that have separate Curie (Néel) temperatures. Thus, this magnetostructural transition with large symmetry change and atomic displacement can bring about various magnetoresponsive effects.

Since the discovery of magnetic field-induced shape memory effect in the Ni-Mn-In Heusler alloy ${ }^{7}$, attempts have been made to induce the magnetostructural transition by applying a magnetic field. To this end, a large magnetization difference $\Delta M$ between the austenite and the martensite phase is necessary to maximize the magnetic-energy change introduced by applying a magnetic field. In a given system, if the MT is tuned to convert the magnetic states from the paramagnetic $(\mathrm{PM})$ to the ferromagnetic $(\mathrm{FM})$ state, rather than from $\mathrm{FM}$ to $\mathrm{FM}^{28}$, a large $\Delta M$ will be gained for the magneticfield-induced MT. With this transition, also a decrease of the magnetic entropy is associated. For a martensitic-transition system, this PM-FM-type MT requires a primary condition that the Curie temperature of the martensite should be higher than that of the austenite. Such an MT is rarely observed in the case of Fe-based and Heusler alloys. Therefore, it is of interest to find an alloy system, which exhibits this particular magnetostructural transition, especially in a broad temperature range.

Recently, the magnetic-field-induced shape memory effect based on the magnetostructural coupling has also been found in another type of materials ${ }^{28}$, the hexagonal ternary compounds with the $\mathrm{Ni}_{2}$ In structure ${ }^{29-31}$. With the FMMTs in these materials, large magnetocaloric effects are also associated ${ }^{32-36}$. In these compounds the magnetic-ordering (Curie or Néel) temperatures of the martensite are higher than those of the austenite ${ }^{32,33,36}$. This satisfies the primary condition for potential PM-FM-type magnetostructural transitions. This large material pool provides a new platform for the desired magnetostructural transitions.

In this study we realize a PM-FM-type magnetostructural transition in hexagonal phase-transition materials in a broad temperature window by suitable chemical substitution of $\mathrm{Fe}$ in MnNiGe. A stable magnetostructural coupling can be achieved by simultaneous manipulation of the phase stability and the magnetic structure. On the basis of valence-electron localization function (ELF) calculations and magnetic configuration analysis, the increased phase stability and the conversion of magnetic structure are, respectively, attributed to the strengthened covalent bonding and $\mathrm{Fe}-6 \mathrm{Mn}$ local atomic configurations introduced by the Fe substitution. The MnNiGe:Fe materials exhibit magnetic-fieldinduced martensitic transitions and giant magnetocaloric effects with broad tunability.

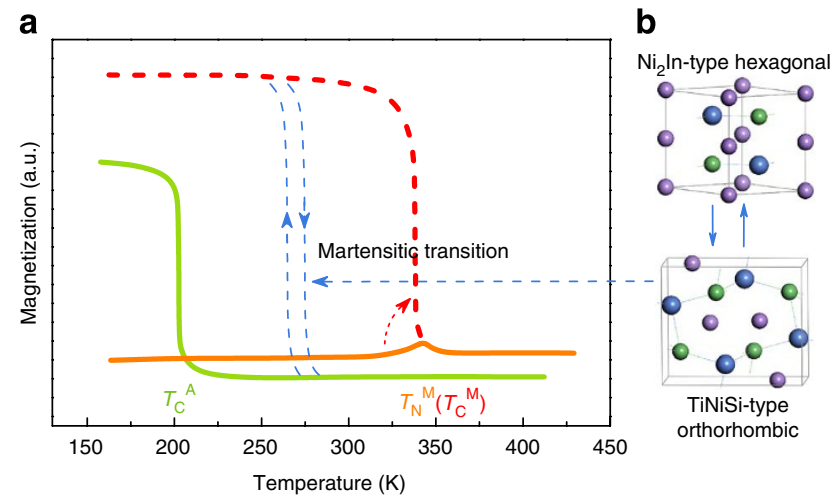

Figure 1 | Expected magnetostructural coupling. (a) Potential temperature window. The light-green and orange lines illustrate the magnetization of the ferromagnetic hexagonal austenite with Curie temperature $\left(T_{C}^{A}\right)$ and the magnetization of the AFM orthorhombic martensite with Néel temperature $\left(T_{N}^{M}\right)$, respectively. There is a broad temperature interval between $T_{C}^{A}$ and $T_{N}^{M}$. (b) MT from Ni 2 In-type hexagonal to TiNiSi-type orthorhombic structure. In stoichiometric MnNiGe, the MT occurs at $T_{\mathrm{t}}=470 \mathrm{~K} \cdot \mathrm{In}(\mathbf{a})$, the dashed blue arrow indicates the expected decrease of $T_{\mathrm{t}}$ and the dashed red arrow the expected AFM to FM conversion. Within the temperature window between $T_{C}^{A}$ and $T_{C}^{M}$, the MT will be coupled with a magnetic transition from the PM to the FM state (indicated by the two dashed blue lines with temperature hysteresis).

\section{Results}

Design scheme. To obtain the desired magnetostructural transition, we consider the hexagonal material $\mathrm{MnNiGe}$ as our starting system. Stoichiometric MnNiGe undergoes an MT at a quite high temperature $T_{\mathrm{t}}=470 \mathrm{~K}$ from the ordered $\mathrm{Ni}_{2}$ In-type hexagonal structure $\left(\mathrm{Pb}_{3} / \mathrm{mmc}, 194\right)$ to the TiNiSi-type orthorhombic structure (Pnma, 62) (refs 37-39; Fig. 1). Because this transition occurs in the PM state, the expected magnetostructural coupling cannot be established. Upon cooling, the martensite phase shows a magnetic transition from the PM state to the antiferromagnetic (AFM) state at a Néel temperature $T_{\mathrm{N}}^{\mathrm{M}}=346 \mathrm{~K}$ (ref. 37). The magnetic moments of $2.8 \mu_{\mathrm{B}}$, which are only localized on the Mn atoms, form an AFM spiral structure 37,38 so that the magnetization is very low. Besides, on the basis of data collected for near-stoichiometric MnNiGe systems (Supplementary Table S1), it can be estimated that the Curie temperature $\left(T_{\mathrm{C}}^{\mathrm{A}}\right)$ of the high-temperature austenite of stoichiometric $\mathrm{MnNiGe}$ lies around $205 \mathrm{~K}$, which is about $140 \mathrm{~K}$ lower than $T_{\mathrm{N}}^{\mathrm{M}}$ of the low-temperature martensite (Fig. 1a). Therefore, MnNiGe shows a potential possibility for the expected PM-FM-type magnetostructural transitions.

For modifying MnNiGe into a desired material, two important changes still have to be introduced in the system. In the first place, the martensitic-transition temperature $T_{t}$ should be lowered in a controllable fashion to a temperature within the temperature interval, as indicated by dotted blue arrow in Fig. 1a, to establish the magnetostructural coupling. The second necessary modification is that the AFM transition in the martensite phase should be converted into a FM transition, that is, $T_{\mathrm{N}}^{\mathrm{M}}$ should become $T_{\mathrm{C}}^{\mathrm{M}}$. This modification is indicated in Fig. la by the dotted red arrow and line. Achievement of these two modifications turns out to be crucial for opening a temperature window between $T_{\mathrm{C}}^{\mathrm{A}}$ and $T_{\mathrm{C}}^{\mathrm{M}}$ in which the PM-FM-type magnetostructural transition with an appreciable value of $\Delta M$ can be realized.

To achieve this, it seems promising to substitute in MnNiGe the magnetic element Fe for the non-magnetic Ni or the magnetic Mn. This is promising because, there is no MT occurring in the isostructural compounds $\mathrm{MnFeGe}$ and FeNiGe so that the austenite structure is maintained down to $4.2 \mathrm{~K}$ (ref. 29). At the same time, the 

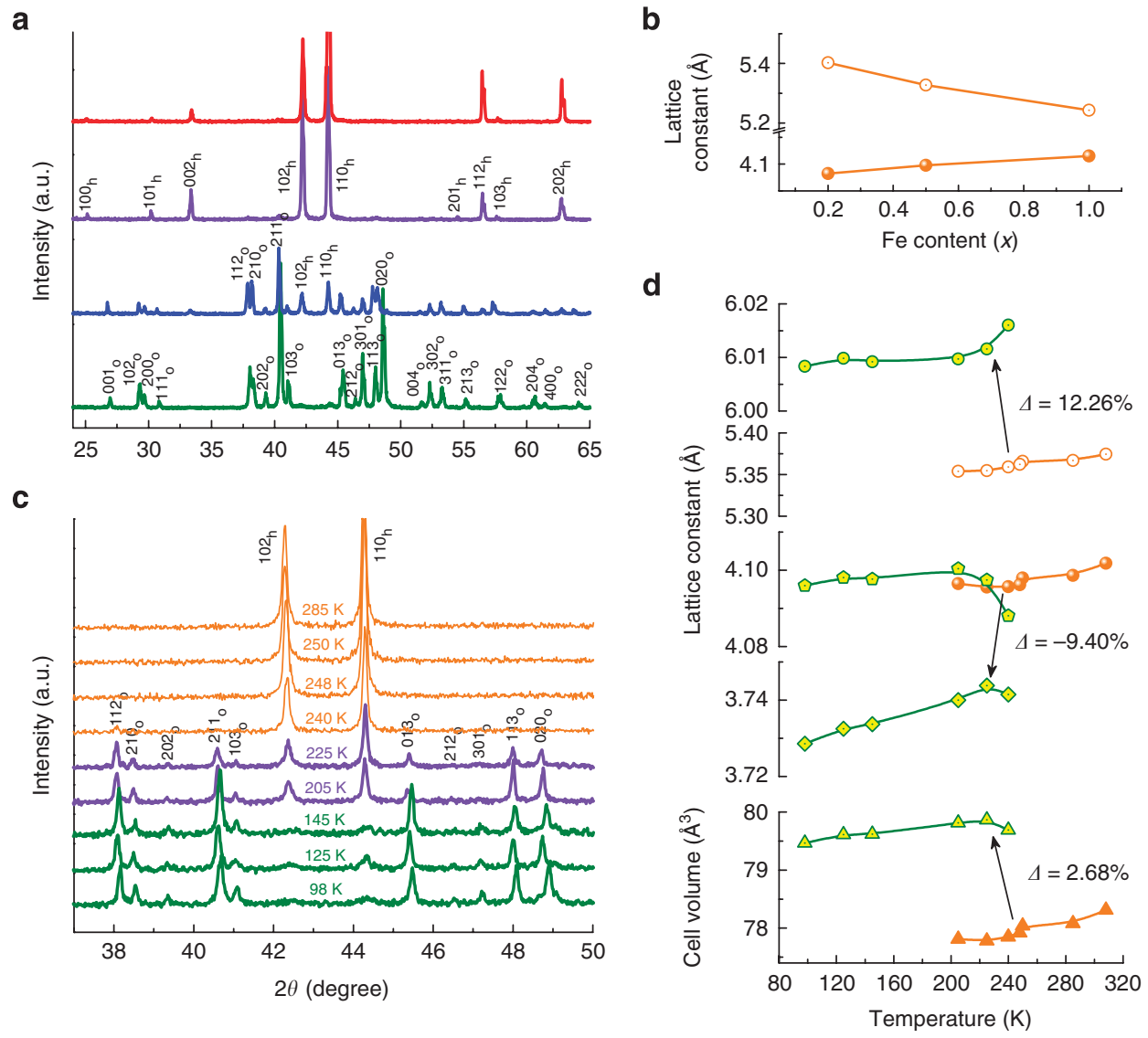

Figure 2 | Structural analysis of the phase transitions. The subscripts ' $h$ ' and ' $o$ ' denote the hexagonal and the orthorhombic structure, respectively. The axes and volumes of the two structures are related as $a_{0}=c_{h}, b_{0}=a_{h}, c_{0}=\sqrt{3} a_{h}$ and $V_{0}=2 V_{h}$ (ref. 39). (a) Composition-dependent XRD of $\mathrm{MnNi}_{1-x} \mathrm{Fe}_{x} \mathrm{Ge}$ at room temperature. Displayed patterns correspond to the Fe contents $x=0$ (green), 0.20 (blue), 0.23 (purple) and 0.27 (red). (b) Variation of the $a_{\mathrm{h}}$ $\left(c_{h}\right)$ axis of $\mathrm{MnNi}_{1-x} \mathrm{Fe}_{x} \mathrm{Ge}$ with Fe content. (c) Temperature-dependent XRD of $\mathrm{Mn}_{0.84} \mathrm{Fe}_{0.16} \mathrm{NiGe}$ from 285 to $98 \mathrm{~K}$, indicating the MT from hexagonal to orthorhombic structure. (d) Temperature-dependent lattice constant and cell volume of $\mathrm{Mn}_{0.84} \mathrm{Fe}_{0.16} \mathrm{NiGe}$ across the MT. The orange and green symbols in (b,d) correspond to the austenite and martensite phases, respectively. The open and solid orange circles in (b,d) stand for $c_{\mathrm{h}}$ and $a_{\mathrm{h}}$ axes, respectively. The green circles, green pentagons and green diamonds in (d) represent the lattice constants $a_{0}, c_{0} / \sqrt{3}$ and $b_{0}$, respectively. The solid and open orange triangles in (d) represent the volumes $V_{\mathrm{h}}$ and $V_{\mathrm{o}} / 2$, respectively.

magnetic Fe may alter the spirally AFM coupling of Mn moments in alloyed MnNiGe. In this sense, alloying these Fe-containing isostructures with $\mathrm{MnNiGe}$ may give rise to a more stable austenite (that is, with lower $T_{\mathrm{t}}$ ) and a higher magnetization of martensite (that is, FM instead of AFM martensite). In the present investigation, we have partly substituted $\mathrm{Fe}$ for $\mathrm{Ni}$ and $\mathrm{Fe}$ for $\mathrm{Mn}$ in $\mathrm{MnNiGe}$ to create the quasi-ternary systems $\mathrm{MnNi}_{1-x} \mathrm{Fe}_{x} \mathrm{Ge}$ and $\mathrm{Mn}_{1-x} \mathrm{Fe}_{x} \mathrm{NiGe}$.

Sample preparation and characterization. The samples were prepared by arc melting and homogenization annealing. The structure of samples was determined with X-ray diffraction (XRD) and no impurity phase was found. Details of the methods are given in the Methods section. With increasing Fe content, the transformation temperature from $\mathrm{Ni}_{2}$ In-type hexagonal austenite to TiNiSi-type orthorhombic martensite is gradually lowered from higher temperatures to below the room temperature (Fig. 2a). The XRD data show that the $c_{\mathrm{h}}\left(a_{\mathrm{h}}\right)$ axis of the austenite phase decreases (increases) upon Fe substitution (Fig. 2b). Temperature-dependent XRD reveals that the MT begins at $240 \mathrm{~K}$ in $\mathrm{Mn}_{0.84} \mathrm{Fe}_{0.16} \mathrm{NiGe}$ (Fig. 2c). An increase of $2.68 \%$ in unit-cell volume is found at the transition (Fig. $2 \mathrm{~d}$; Supplementary Table S2). This volume expansion is large and opposite to the usual contraction of about $-1 \%$ at martensitic structural transitions. This indicates that the crystalline structure and the atomic surrounding undergo a pronounced reconstruction during the structural transition, as shown in Figure 1b.
Structural and magnetic phase diagrams. To determine the crystallographic and magnetic structures, low- and high-field $M(T)$ measurements and differential thermal analysis (DTA) were used (Supplementary Figs. S1 and S2; Table 1). On the basis of the experimental data, the $\mathrm{MnNi}_{1-x} \mathrm{Fe}_{x} \mathrm{Ge}$ and $\mathrm{Mn}_{1-x} \mathrm{Fe}_{x} \mathrm{NiGe}$ phase diagrams are proposed as shown in Fig. 3. In both systems, the $\mathrm{Fe}$ substitution makes the MT fall within the temperature range of ferromagnetic order of martensites.

Upon substitution of Fe for $\mathrm{Ni}\left(\mathrm{MnNi}_{1-x} \mathrm{Fe}_{x} \mathrm{Ge}\right.$, Fig. $\left.3 \mathrm{a}\right), T_{\mathrm{N}}^{\mathrm{M}}$ becomes $T_{\mathrm{C}}^{\mathrm{M}}$ at about $300 \mathrm{~K}$. Upon further increase of Fe content, $T_{\mathrm{t}}$ continuously decreases until $T_{\mathrm{C}}^{\mathrm{A}}$ is reached. It can also be seen that, upon substitution, both $T_{\mathrm{N}}^{\mathrm{M}}\left(T_{\mathrm{C}}^{\mathrm{M}}\right)$ and $T_{\mathrm{C}}^{\mathrm{A}}$ basically remain constant, which offers an accessible temperature window of about $90 \mathrm{~K}$ between them. Within this window, the system undergoes an MT coupled with a magnetic transition from the PM to the FM state. Below $T_{\mathrm{C}}^{\mathrm{A}}$, the magnetostructural transition decouples as the MT rapidly vanishes. In the case of substitution of Fe for $\mathrm{Mn}\left(\mathrm{Mn}_{1-x} \mathrm{Fe}_{x} \mathrm{NiGe}\right.$, Fig. 3b), a quite low level of Fe substitution (about $x=0.08$ ) already lowers $T_{\mathrm{t}}$ to meet $T_{\mathrm{N}}^{\mathrm{M}}$ and to introduce ferromagnetism at a relatively high temperature of $350 \mathrm{~K}$. In the range $0.08 \leq x \leq 0.26$, the FM martensite phase has a high magnetization in $5 \mathrm{~T}$. The Fe substitution efficiently converts AFM martensite into FM one while, surprisingly, it still drives the FM austenite parent phase into a weak-magnetic spin-glass-like state (Fig. 4; Supplementary Fig. S3). Along with the eventual vanishing of the MT at the freezing temperature $\left(T_{\mathrm{g}}\right)$ of 
Table 1 | Key parameters of MnNiGe:Fe systems.

\begin{tabular}{|c|c|c|c|c|c|c|c|c|c|c|c|}
\hline \multirow[t]{2}{*}{ Alloying case } & \multirow[t]{2}{*}{$x$} & $\begin{array}{c}T_{\mathbf{t}} \\
\text { (cooling)* }\end{array}$ & 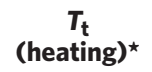 & $\Delta T r$ & $T_{C}^{A}\left(T_{g}\right) \div$ & $T_{N} M_{S}$ & $\mathbf{M}^{\mathbf{M}} \|$ & $\mathbf{M}^{\mathbf{A} \text { ๆ }}$ & $\Delta \mathbf{M \#}$ & $B_{\mathrm{cr}^{\star \star}}$ & $B_{s}+t$ \\
\hline & & \multicolumn{5}{|c|}{$\mathbf{K}$} & \multicolumn{3}{|c|}{$A m^{2} k g^{-1}$} & \multicolumn{2}{|c|}{ Tesla } \\
\hline \multirow{12}{*}{$\mathrm{MnNi}_{1-x} \mathrm{Fe}_{x} \mathrm{Ge}$} & 0 & - & 470t+ & - & - & $346+t$ & $39+4$ & - & - & - & $>5+t$ \\
\hline & & 460 & 508 & 48 & - & 352 & 46 & - & - & 1.20 & $>5$ \\
\hline & 0.10 & 400 & 416 & 16 & - & 324 & 52 & - & - & 0.90 & $>5$ \\
\hline & 0.15 & 352 & 366 & 14 & - & 306 & 72 & - & - & 0.60 & $>5$ \\
\hline & 0.20 & 300 & 312 & 12 & - & 291 & 87 & - & 46 & 0.45 & $>5$ \\
\hline & 0.23 & 266 & 276 & 10 & - & - & 92 & - & 51 & 0.35 & $>5$ \\
\hline & 0.27 & 230 & 240 & 10 & - & - & 95 & - & 49 & 0.20 & $>5$ \\
\hline & 0.30 & 189 & 201 & 12 & 211 & - & 98 & - & 40 & 0.05 & 5.0 \\
\hline & 0.33 & - & - & - & 213 & - & - & 77 & - & - & - \\
\hline & 0.36 & - & - & - & 215 & - & - & 74 & - & - & - \\
\hline & 0.40 & - & - & - & 218 & - & - & 72 & - & - & - \\
\hline & 0.50 & - & - & - & 220 & - & - & 73 & - & - & - \\
\hline \multirow[t]{15}{*}{$\mathrm{Mn}_{1-x} \mathrm{Fe}_{x} \mathrm{NiGe}$} & 0.03 & 429 & 472 & 43 & - & 349 & 52 & - & - & 1.13 & $>5$ \\
\hline & 0.05 & 389 & 409 & 20 & - & 347 & 65 & - & - & 0.86 & $>5$ \\
\hline & 0.08 & 336 & 357 & 21 & - & 341 & 75 & - & 36 & 0.57 & 5.0 \\
\hline & 0.11 & 298 & 305 & 7 & - & - & 78 & - & 55 & 0.48 & 4.0 \\
\hline & 0.13 & 277 & 285 & 8 & - & - & 80 & - & 57 & 0.45 & 3.0 \\
\hline & 0.16 & 241 & 249 & 8 & - & - & 78 & - & 59 & 0.33 & 2.3 \\
\hline & 0.18 & 207 & 214 & 7 & - & - & 79 & - & 59 & 0.24 & 1.8 \\
\hline & 0.22 & 153 & 163 & 10 & - & - & 76 & - & 52 & 0.05 & 0.9 \\
\hline & 0.24 & 127 & 135 & 8 & - & - & 75 & - & 54 & 0 & 0.5 \\
\hline & 0.26 & 74 & 84 & 10 & - & - & - & 25.7 & - & - & - \\
\hline & 0.28 & - & - & - & 80 & - & - & 23.0 & - & - & - \\
\hline & 0.30 & - & - & - & 78 & - & - & 22.3 & - & - & - \\
\hline & 0.35 & - & - & - & 75 & - & - & 17.6 & - & - & - \\
\hline & 0.40 & - & - & - & 70 & - & - & 17.2 & - & - & - \\
\hline & 0.50 & - & - & - & 65 & - & - & 18.1 & - & - & - \\
\hline \multicolumn{12}{|c|}{$\begin{array}{l}{ }^{\star} \text { Martensitic transition temperature. } \\
\dagger \text { Temperature hysteresis of martensitic transition. } \\
\text { †Curie temperature of austenites. } \mathrm{T}_{\mathrm{g}} \text { is frozen temperature of spin-glass-like state of } \mathrm{Mn}_{1-x} \mathrm{Fe} \\
\text { §Néel temperatures of martensites. Determined as average values measured on cooling and } \mathrm{H} \\
\| \text { Martensitic magnetization. Measured at } 5 \mathrm{~K} \text { in a field of } 5 \mathrm{~T} \text {. } \\
\text { TAustenitic magnetization. Measured at } 5 \mathrm{~K} \text { in a field of } 5 \mathrm{~T} \text {. } \\
\text { \#Magnetization difference between austenite and martensite across martensitic transition. } \\
{ }_{\star \star *} \text { Critical field of metamagnetic transition in martensite phase. } \\
\dagger \dagger \text { Saturation field of martensite. } \\
\text { HFrom ref. } 37 \text {. }\end{array}$} \\
\hline
\end{tabular}

the spin-glass-like state, the significant consequence is obtained: the lowest temperature of the window has moved down to about $70 \mathrm{~K}$ and a quite broad temperature interval up to $280 \mathrm{~K}$ is generated for the stable magnetostructural coupling.

It should be further pointed out that, the temperature hysteresis of the MT for both systems is significantly reduced from about $50 \mathrm{~K}$ to below $10 \mathrm{~K}$ by the Fe substitution (Fig. $3 \mathrm{c}$ and d; Table 1), which implies a decreasing thermodynamic driving force for the martensite nucleation. For a first-order MT, the hysteresis of $10 \mathrm{~K}$ is very small $^{40}$, which is beneficial for the temperature sensibility of magnetoresponsive smart applications based on martensitic transitions.

Thermomagnetic behaviour. To clarify the PM-FM MTs in the broad window, we measured the high-field thermomagnetic properties of the typical samples of $\mathrm{Mn}_{1-x} \mathrm{Fe}_{x} \mathrm{NiGe}$ system, as shown in Figure 4 (thermomagnetic properties of $\mathrm{MnNi}_{1-x} \mathrm{Fe}_{x} \mathrm{Ge}$ system are shown in Supplementary Fig. S4). In accordance with Fig. 3, $T_{\mathrm{t}}$ decreases with increasing Fe content. For $x>0.08$, PM-FM jumps of the magnetization, with large $\Delta M$ up to $60 \mathrm{~A} \mathrm{~m}^{2} \mathrm{~kg}^{-1}$ in a field of $5 \mathrm{~T}$, are observed. This signifies that the introduction of Fe has led to a great change of the magnetic exchange interaction in the martensite phase, changing the spiral AFM structure into a FM state. Upon cooling, for each composition the FM martensite phase nucleates and grows in the PM austenite matrix. Upon heating, the reversible nature of the MT can be seen. Here, it should be emphasized again that $T_{\mathrm{t}}$ is the martensitic-transition temperature, not the Curie temperature. The Curie (Néel) temperatures of both phases have the values at the respective window boundaries, which are shown in Fig. 3b. When the transition occurs, the austenite is still in PM state, whereas the martensite is already in its FM state. It is the crystallographic structural transition between a PM phase and a FM phase that gives rise to the abrupt magnetization change. For $x=0.26$, it can be seen that the MT becomes incomplete and the spin-glass-like behaviour shown by the irreversible zero-field-cooling/field-cooling (ZFC-FC) curves is in accordance with the phase diagram in Figure 3b. Within this broad temperature window, a stable magnetostructural coupling is obtained from above room temperature $(350 \mathrm{~K})$ to liquid-nitrogen temperature $(70 \mathrm{~K})$.

Magnetic field-induced properties across the transitions. In what follows, we study typical magnetoresponsive properties for both systems. First, we present the magnetic-field-induced MT effect. Fig. 5a shows the magnetization curves of $\mathrm{MnNi}_{0.77} \mathrm{Fe}_{0.23} \mathrm{Ge}$ at various temperatures within the temperature window. Above $276 \mathrm{~K}$, the austenite shows a PM behaviour. Between 274 and $258 \mathrm{~K}$, the continuous metamagnetic behaviour at each temperature in high field (marked by arrows) reveals a field-induced MT effect, indicating that the FM martensite phase is induced by an applied field in the PM austenite matrix. This behaviour corresponds to an upward shift of about $11 \mathrm{~K}$ of the martensitic starting transition 


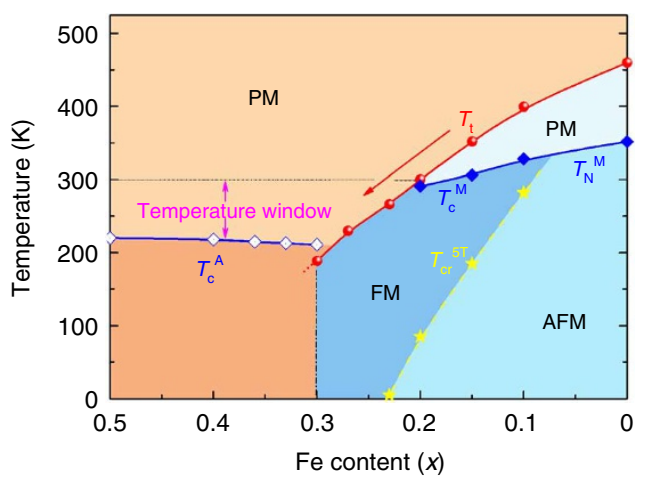

C

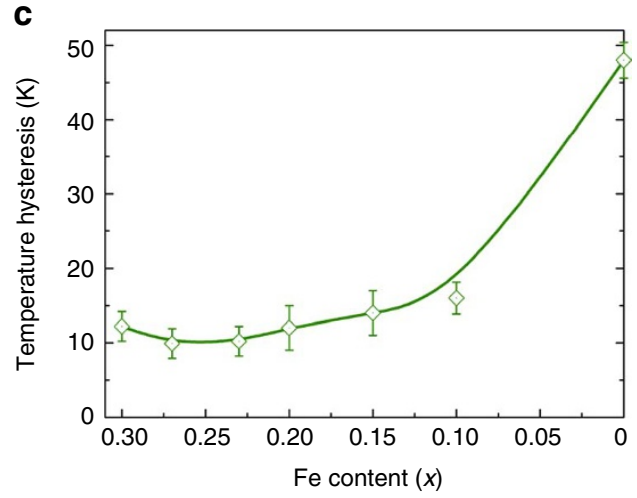

b
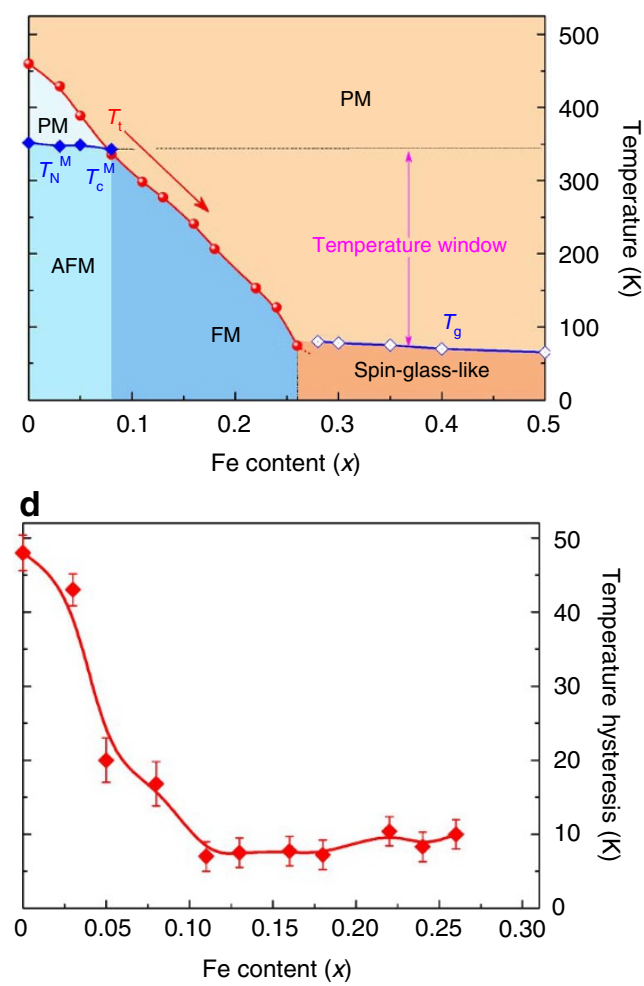

Figure 3 | Structural and magnetic phase diagrams and phase-transition hysteresis. PM, FM and AFM indicate the PM austenite, the ferromagnetic

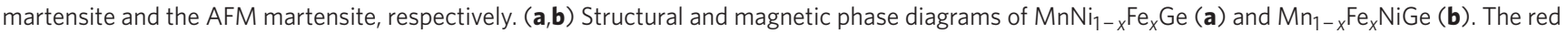
circles denote the martensitic-transition temperature $T_{\mathrm{t}}$ and the red arrows indicate the decreasing trend of $T_{\mathrm{t}}$. In the range $0 \leq x \leq 0.30$ for $\mathrm{MnNi}_{1}-\mathrm{Fe}_{x} \mathrm{Ge}_{\mathrm{f}}$ (a) and $0 \leq x \leq 0.26$ for $\mathrm{Mn}_{1-x} \mathrm{Fe}_{x} \mathrm{NiGe}(\mathbf{b})$, the systems undergo a Ni 2 In-type to TiNiSi-type MT at $T_{\mathrm{t}}$. Above $x=0.30$ (a) and $x=0.26$ (b), the systems are single-phase $\mathrm{Ni}_{2}$ In-type austenites. The solid diamonds correspond to $T_{N}^{\mathrm{M}}\left(T_{\mathrm{C}}^{\mathrm{M}}\right)$ of the martensite $(\mathbf{a}, \mathbf{b})$ and the open diamonds to $T_{\mathrm{C}}^{\mathrm{A}}(\mathbf{a})$ and $T_{g}(\mathbf{b})$ of the austenite. In the range $0.20 \leq x \leq 0.30(\mathbf{a})$ and $0.08 \leq x \leq 0.26(\mathbf{b})$, the temperature windows are limited by $T_{C}^{M}-T_{C}^{A}(\mathbf{a})$ and by $T_{C}^{M}-T_{g}(\mathbf{b})$, respectively. In (a), the FM state of the martensite returns back to an AFM state upon cooling. The yellow stars show the critical temperature ( $T_{c r}^{5 T}$ ) between the FM and AFM states in a field of 5 T (more details in Supplementary Fig. S4). In (b), the austenitic-phase zone with $\times \geq 0.26$ enters into a spin-glass-like state below about $70 \mathrm{~K}$ (more details in Supplementary Fig. S3). (c,d) Temperature hysteresis of the first-order martensitic transition for $\mathrm{MnNi}_{1-x} \mathrm{Fe}_{x} \mathrm{Ge}_{\text {(c) }}$ and $\mathrm{Mn}_{1-x} \mathrm{Fe}_{x} \mathrm{NiGe}(\mathbf{d})$. Here, the hysteresis is defined as the interval of peak values of the $\mathrm{dM} / \mathrm{dT}$ and/or DTA curves upon cooling and upon heating (Supplementary Figs S1, S2; Table 1). The error bars of the hysteresis in (c,d) are given by repeating the measurements.

temperature by a field of $5 \mathrm{~T}$ (more details in Supplementary Fig. S5), which means that the martensite phase appears at a higher temperature with the aid of the magnetic field. On the basis of the AFM-FM conversion of the martensite upon Fe substitution, the appreciable $\Delta M$ between the austenite and martensite introduces a larger Zeeman energy for the martensite in the magnetic field, giving rise to energetically more favourable martensite. Similarly, a more distinct PM-FM filed-induced MT also occurs in $\mathrm{Mn}_{0.82} \mathrm{Fe}_{0.18} \mathrm{NiGe}$, as shown in Fig. 5b. On the basis of this fieldinduced MT effect, magnetic-field-controlled ferromagnetic shape memory alloys may be prepared in MnNiGe:Fe system. Moreover, this MT with a large volume increase (Fig. 2d) implies the volume of the material can be significantly changed by an applied field. This may benefit the magnetic-field-induced strains for potential strain-based applications.

Associated with the sharp first-order magnetostructural transition, a magnetic-entropy change $\left(\Delta S_{\mathrm{m}}\right)$ occurs $^{41,42}$. By means of the Maxwell relation and loop process method (see Methods), the magnetic-entropy changes at the transitions have been derived from the magnetization curves of $\mathrm{MnNi}_{1-x} \mathrm{Fe}_{x} \mathrm{Ge}(x=0.23)$ (Fig. 5c) and $\mathrm{Mn}_{1-x} \mathrm{Fe}_{x} \mathrm{NiGe}(x=0.18)$ (Fig. 5d). Because the low-temperature martensite is FM and the high-temperature austenite is PM $(\partial M / \partial T<0)$, all samples exhibit a negative $\Delta S_{\mathrm{m}}$. In the temperature window, $\mathrm{MnNi}_{0.77} \mathrm{Fe}_{0.23} \mathrm{Ge}$ exhibits a large $\Delta S_{\mathrm{m}}$ value of $-19 \mathrm{~J} \mathrm{~kg}^{-1} \mathrm{~K}^{-1}$ for $\Delta B=5 \mathrm{~T}$ (Fig. $5 \mathrm{c}$ ). This window offers the possibility to obtain large $\Delta S_{\mathrm{m}}$ values for the $\mathrm{MnNi}_{1-x} \mathrm{Fe}_{x} \mathrm{Ge}$ system in an interval of nearly $100 \mathrm{~K}$. In the $\mathrm{Mn}_{1-x} \mathrm{Fe}_{x} \mathrm{NiGe}$ system, even more appreciable $\Delta S_{\mathrm{m}}$ values are observed in an even more extended temperature window ranging from 350 to $70 \mathrm{~K}$. As an example, a low substitution level of $x=0.18$ gives rise to a giant $\Delta S_{\mathrm{m}}$ value of $-31 \mathrm{Jkg}^{-1} \mathrm{~K}^{-1}$ for $\Delta B=5 \mathrm{~T}$ (Fig. $5 \mathrm{~d}$ ). These larger $\Delta S_{\mathrm{m}}$ values are attributed to the more ferromagnetically ordered martensite and thus a lower magnetic-entropy state after the transition. In accordance with the field-induced MT effect, the $\Delta S_{\mathrm{m}}$ peak position also shows a field dependence and shifts to higher temperatures with increasing magnetic field (dashed grey lines in Fig. $5 \mathrm{c}$ and d). Another feature of the MnNiGe:Fe systems is that the magnetic and martensitic transitions have the same sign of the enthalpy change (more details in Supplementary Figs S1 and S2), as the crystallographic and magnetic symmetries are both lowered on cooling. This prevents opposite heat processes that counteract the caloric effects, which is very different from the common FM-AFM(PM) MTs in Fe-based and Heusler ferromagnetic shape memory alloys.

\section{Discussion}

In this study, stable magnetostructural coupling has been realized by appropriate material design and the associated magnetoresponsive effects have been presented. This magnetostructural coupling has been achieved by decreasing $T_{\mathrm{t}}$ of MT of the alloyed MnNiGe and converting the AFM to the FM state in martensite phase by 


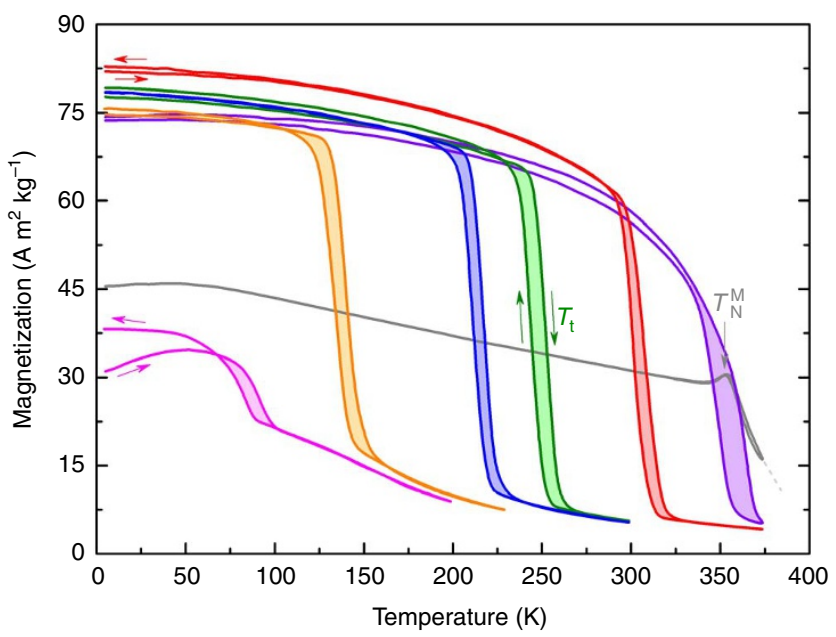

Figure 4 | Magnetostructural coupling in the established temperature window. The magnetization curves of $\mathrm{Mn}_{1-x} \mathrm{Fe}_{x} \mathrm{NiGe}$ have been measured at ZFC-FC in an applied magnetic field of $5 \mathrm{~T}$. The grey curve represents the magnetization of the spiral AFM martensite of Fe-free MnNiGe. For this stoichiometric $\mathrm{MnNiGe}, T_{\mathrm{N}}^{\mathrm{M}}$ is $352 \mathrm{~K}$ and $T_{\mathrm{t}}(460 \mathrm{~K})$ is outside the temperature window. The magnetostructural coupling based on the PMFM-type MT is obtained in a broad temperature window. Displayed curves correspond to Fe contents $x=0.08$ (purple), 0.11 (red), 0.16 (green), 0.18 (blue), 0.24 (orange) and 0.26 (pink). An incomplete MT and spin-glasslike behaviour are observed for the sample with $x=0.26$ (more details in Supplementary Fig. S3). replacing $\mathrm{Ni}$ or $\mathrm{Mn}$ by $\mathrm{Fe}$. We will discuss their possible origins in this section.

As mentioned before, the isostructural compounds MnFeGe and FeNiGe have a stable $\mathrm{Ni}_{2}$ In-type austenite structure without MT. This reasonably causes the decrease of the structural transition temperature $T_{\mathrm{t}}$ (that is, the increasingly stable austenite phase) upon substitution of Fe in MnNiGe. Inherently, this is related to the strengthening of local chemical bonds when Fe atoms are introduced at Ni or Mn sites. To get an insight into the change of the chemical bonds, we have calculated the ELF (Becke and Edgecombe ${ }^{43}$, see Methods) for $\mathrm{MnNi}_{0.5} \mathrm{Fe}_{0.5} \mathrm{Ge}$ (Fig. 6). In this highly ordered substituted structure, an alternating sequence of $\mathrm{Fe}-\mathrm{Ge}$ and $\mathrm{Ni}-\mathrm{Ge}$ layers is formed (Fig. 6a,b), which provides a convenient comparison between $\mathrm{Fe}-\mathrm{Ge}$ and Ni-Ge bonding. From the topological analysis of ELF, it can be seen that the electron localization between nearest-neighbor Fe and $\mathrm{Ge}$ atoms ( $\mathrm{Fe}-\mathrm{Ge} 1$ and $\mathrm{Fe}-\mathrm{Ge} 2)$ is strengthened with respect to that between nearest-neighbor $\mathrm{Ni}$ and $\mathrm{Ge}$ atoms (Ni-Ge1 and $\mathrm{Ni}-\mathrm{Ge} 2)$. Meanwhile, Fe-substitution leads to a remarkable reduction (from 2.749 to $2.579 \AA$ ) of the distance between $\mathrm{Mn}$ atoms on each side of $\mathrm{Fe}-\mathrm{Ge}$ layer, which is in agreement with the reduction of the $c_{\mathrm{h}}$ axis in the XRD results (Fig. 2b). Thus, an ELF basin between the Mn2$\mathrm{Mn} 3$ atoms also appears (in orange colour), which, in contrast, is absent between Mn1-Mn2 atoms. These results indicate that substitution of Fe for $\mathrm{Ni}$ in MnNiGe can lead to strengthening of the covalent bonding between neighbouring $\mathrm{Fe}$ and $\mathrm{Ge}$ atoms and between neighbouring $\mathrm{Mn}$ and $\mathrm{Mn}$ atoms, which is thus largely responsible for the stabilization of the high-temperature austenite phase.

Next, we should address the origin of the conversion of the AFM to the FM state in the alloyed martensite. In the martensite phase of
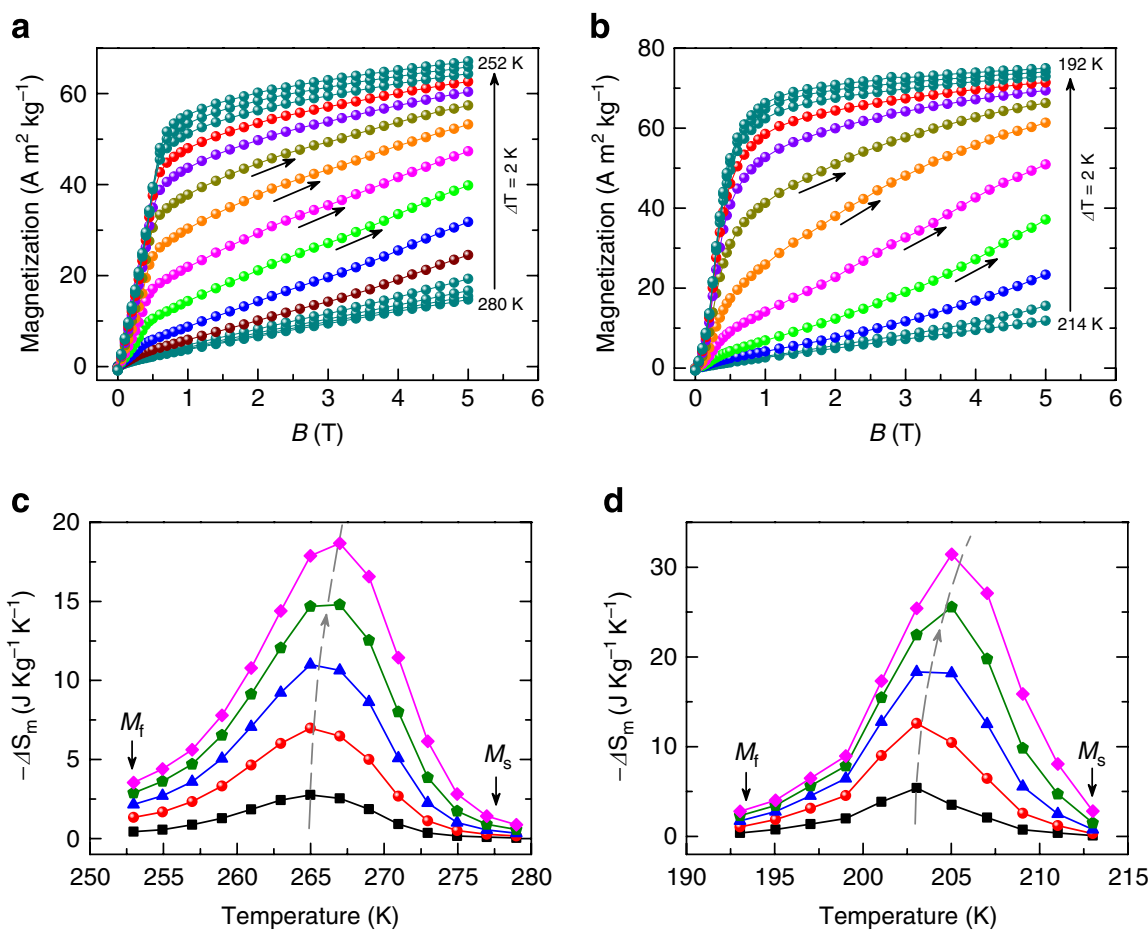

Figure 5 | Magnetic-field-induced martensitic transitions and magnetic-entropy changes. (a,b) Magnetic isotherms of $\mathrm{MnNi}_{0.77} \mathrm{Fe}_{0.23} \mathrm{Ge}_{(\mathbf{a})}$ and $\mathrm{Mn}_{0.82} \mathrm{Fe}_{0.18} \mathrm{NiGe}(\mathbf{b})$ at various temperatures in the temperature window. The measurement temperature deceases from 280 to $252 \mathrm{~K}$ (a) and from 214 to $192 \mathrm{~K}$ (b) with an interval of $2 \mathrm{~K}$. The metamagnetic behaviour (marked by arrows) indicates the magnetic-field-induced martensitic transition. (c, d) Isothermal magnetic-entropy changes $\left(\Delta S_{m}\right)$ for various field changes derived from the magnetic isotherms of $\mathrm{MnNi}_{1}-\mathrm{Fe}_{x} \mathrm{Ge}(\mathbf{c})$ and $\mathrm{Mn}_{1-x} \mathrm{Fe}_{\chi} \mathrm{NiGe}$ (d). Displayed curves correspond to the field changes $\Delta B=1 \mathrm{~T}$ (black squares), $2 \mathrm{~T}$ (red circles), $3 \mathrm{~T}$ (blue triangles), $4 \mathrm{~T}$ (green pentagons) and $5 \mathrm{~T}$ (pink diamonds). The shift of the $\Delta S_{\mathrm{m}}$ maximum with increasing field to higher temperatures (indicated by the dashed grey lines) is related to the magnetic-field-induced MT effect. $M_{\mathrm{s}}$ and $M_{\mathrm{f}}$ denote the starting and finishing temperatures of the MT, respectively. 
a

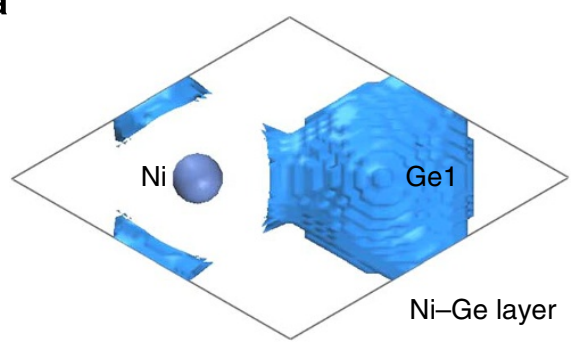

b

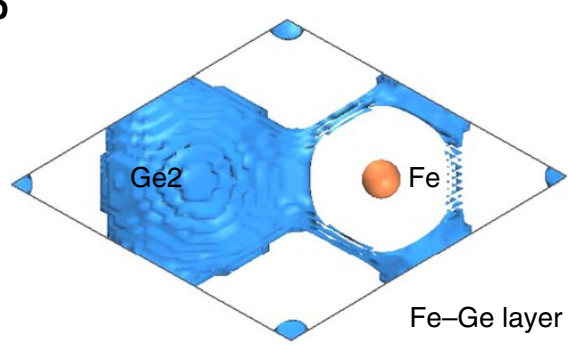

C

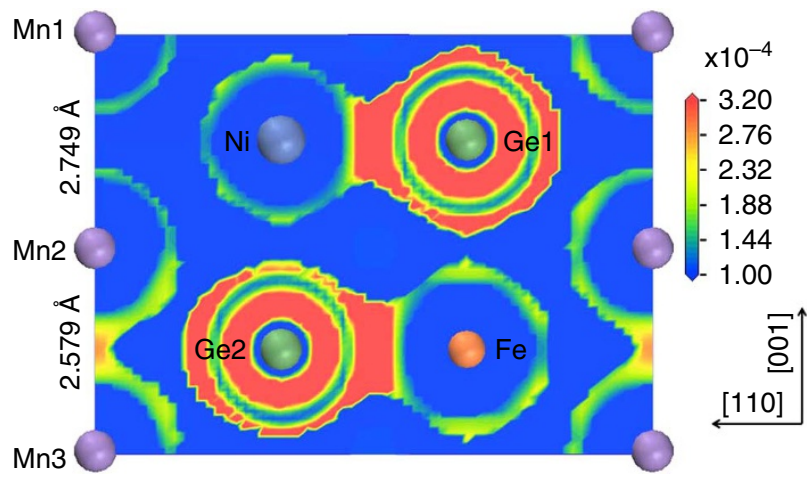

Figure 6 | ELF of $\mathbf{M n N i}_{0.5} \mathbf{F e}_{0.5} \mathbf{G e}$ austenite. $(\mathbf{a}, \mathbf{b})$ ELF isosurface basins (top view) with an isovalue of $2.35 \times 10^{-4}$ for $\mathrm{Ni}-\mathrm{Ge}$ (a) and $\mathrm{Fe}-\mathrm{Ge}(\mathbf{b})$ layers. The $\mathrm{Ge} 1$ and $\mathrm{Ge} 2$ atoms are encapsulated by separate isosurfaces. More surfaces appear around Fe than around Ni. (c) ELF contour map in the (110) plane. The scale bar from blue to red corresponds to increasing electron localization (namely, increasing covalent bonding). Compared with $\mathrm{Ni}-\mathrm{Ge} 1$ and $\mathrm{Ni}-\mathrm{Ge} 2$, there are higher values at $\mathrm{Fe}-\mathrm{Ge} 2$ and $\mathrm{Fe}-\mathrm{Ge} 1$. The distance between $\mathrm{Mn} 2$ and $\mathrm{Mn} 3$ atoms at different sides of the Fe-Ge layer is reduced compared with that between $\mathrm{Mn} 1$ and $\mathrm{Mn} 2$ atoms at different sides of the Ni-Ge layer.

stoichiometric $\mathrm{MnNiGe}$ compound ${ }^{38}$, the zero-moment $\mathrm{Ni}$ atoms are surrounded by six nearest-neighbour Mn atoms, forming a local $\mathrm{Ni}-6 \mathrm{Mn}$ configuration (Fig. 7a, light-blue zone; more details in Supplementary Fig. S6). The spiral AFM structure originates from the specific exchange interactions in the Mn moments in this specific moment and lattice configurations in the matrix. In high applied magnetic fields, this spiral AFM structure changes into a canted FM structure, which eventually saturates ferromagnetically at about $10 \mathrm{~T}$ (ref. 37). This behaviour illustrates the instability of the spiral AFM magnetic structure in the stoichiometric MnNiGe martensite.

According to the atomic occupancy rule in ordered $\mathrm{Ni}_{2} \mathrm{In}$ type compounds (more details in Supplementary Fig. S6), Fe in $\mathrm{MnNi}_{1-x} \mathrm{Fe}_{x} \mathrm{Ge}$ simply occupies the $\mathrm{Ni}$ sites. Similarly, Fe in $\mathrm{Mn}_{1-x} \mathrm{Fe}_{x} \mathrm{NiGe}$ occupies the $\mathrm{Mn}$ sites. Here, we take $\mathrm{MnNi}_{1-x} \mathrm{Fe}_{x} \mathrm{Ge}$ system as an example. Owing to the unchanged relative-site occupation during the diffusionless and displacesive MT, all atoms consistently occupy their respective sites after the transition. In Fe-substituted martensite, whichever positions on Ni sites the Fe atoms occupy, some $\mathrm{Ni}-6 \mathrm{Mn}$ local atomic configurations naturally change into Fe-6Mn ones. That is, crystallographically, every introduced $\mathrm{Fe}$ atom is always surrounded by six nearest-neighbour $\mathrm{Mn}$ atoms. With magnetic moments of $0.5<\mu<1 \mu_{\mathrm{B}}$ (ref. 44) for Fe atoms, this Fe-6Mn local configuration internally establishes FM coupling. The spirally AFM-coupled Mn moments within the configuration is thus changed into parallel alignment because of the Fe moments. We schematically illustrate this FM Fe-centered local configuration (pink zones) in comparison with the spiral AFM matrix in Fig. 7a. This mechanism is similar to the FM exchange interaction established between $\mathrm{Mn}$ and Co atoms by substituting Co $\left(\mu=1 \mu_{\mathrm{B}}\right)$ for $\mathrm{Ni}$ in $\mathrm{MnNiGe}$ (ref. 45). With increasing Fe content, the number of FM configurations will increase and they will overlap and form larger FM zones, in this way promoting the AFM-FM conversion in the martensite phase.

The above-described FM coupling in Fe-6Mn configurations is explicitly confirmed by the magnetization behaviour of $\mathrm{MnNi}_{1-x} \mathrm{Fe}_{x} \mathrm{Ge}$ martensites at $5 \mathrm{~K}$ in fields of up to $5 \mathrm{~T}$ (Fig. $7 \mathrm{~b}$ ). The Fe-free sample shows AFM behaviour with a metamagnetic kink at a critical field $B_{\mathrm{cr}}$ of $1.3 \mathrm{~T}$, indicating a metamagnetic transition from a spiral AFM to a canted FM state, in accordance with the reported stoichiometric $\mathrm{MnNiGe}$ (ref. 37). In contrast, the Fe-substituted martensites show a large slope of the $M(B)$ curves in low fields that increases as a function of the Fe content. This suggests that an increasing FM component is generated in the system due to the existence of the Fe-centered Fe-6Mn configurations. Meanwhile, $B_{\text {cr }}$ rapidly decreases with increasing Fe content (Fig. 7b,d), which corresponds to an increasing ease for the applied field to change the spiral AFM structure to a forced parallel alignment. The larger the number of local Fe-6Mn configurations becomes, lower the $B_{\text {cr }}$ will be. For $x=0.30$ (the highest Fe content in the martensite phase due to the vanishing of the MT for higher substitution), a FM groundstate with a very low $B_{\mathrm{cr}}$ of $0.05 \mathrm{~T}$ is found. Owing to both the Fe substitution and the applied field, the magnetization reaches values of up to about $100 \mathrm{~A} \mathrm{~m}^{2} \mathrm{~kg}^{-1}$ in a field of $5 \mathrm{~T}$, much higher than that of the Fe-free sample (Fig. 7b,d).

By substituting Fe for $\mathrm{Mn}$ in MnNiGe, the AFM-FM conversion is expedited (Fig. 7c). This is because the Fe atoms not only introduce FM coupling between Fe and Mn atoms, but also break up the consecutive AFM sublattices of the Mn moments. This rapidly makes the AFM order collapse. Thus, only a small Fe content of about $x=0.08$ is sufficient to approach the maximal magnetization for $\mathrm{Mn}_{1-x} \mathrm{Fe}_{x} \mathrm{NiGe}$ (Fig. 7c,d). The samples more and more easily get magnetically saturated and show a rapidly decreasing saturation field (Fig. 7c,e). The complete FM ground state appears in the sample with $x=0.22$ (Fig. 7c), versus $x=0.30$ in $\mathrm{MnNi}_{1-x} \mathrm{Fe}_{x} \mathrm{Ge}$ (Fig. 7b). Therefore, we conclude that the AFM-FM conversion becomes more efficient when the substituted Fe atoms occupy Mn sites in $\mathrm{Mn}_{1-x} \mathrm{Fe}_{x} \mathrm{NiGe}$. As a consequence, this FM state in martensite phase facilitates the magnetoresponsive effects presented in this study.

In previous studies ${ }^{45,46}$, it has been reported that the large-size $\mathrm{Ge}$ and zero-moment $\mathrm{Ni}$ in $\mathrm{MnNiGe}$ can be replaced by small-size $\mathrm{Si}$ and magnetic Co, respectively. Actually, these replacements are also equal to alloying the isostructural $\mathrm{Ni}_{2}$ In-type $\mathrm{MnNiSi}$ and $\mathrm{MnCoGe}$ compound with the MnNiGe mother compound. MnNiSi and $\mathrm{MnCoGe}$ undergo MTs at high temperatures and their martensites are both ferromagnetic ${ }^{39}$. Therefore, in insert martensite structure, $\mathrm{MnNiSi}$ and $\mathrm{MnCoGe}$ can reasonably change the AFM state of $\mathrm{MnNiGe}$ into a FM state. However, they fail to lower the temperature of MT from $470 \mathrm{~K}$ of $\mathrm{MnNiGe}$ to below $T_{\mathrm{C}}^{\mathrm{M}}$, which would establish the coupling needed for the magnetostructural transition. The substitution of Fe applied in this study thus shows the best results for both the decrease of $T_{\mathrm{t}}$ and the magnetic-state conversion of MnNiGe; that is, for the desired PM-FM magnetostructural transition. Very recently, an interesting paper has been published on the pressure-tuned magnetostructural transition in Cr-doped $\mathrm{MnCoGe}^{47}$. 
a

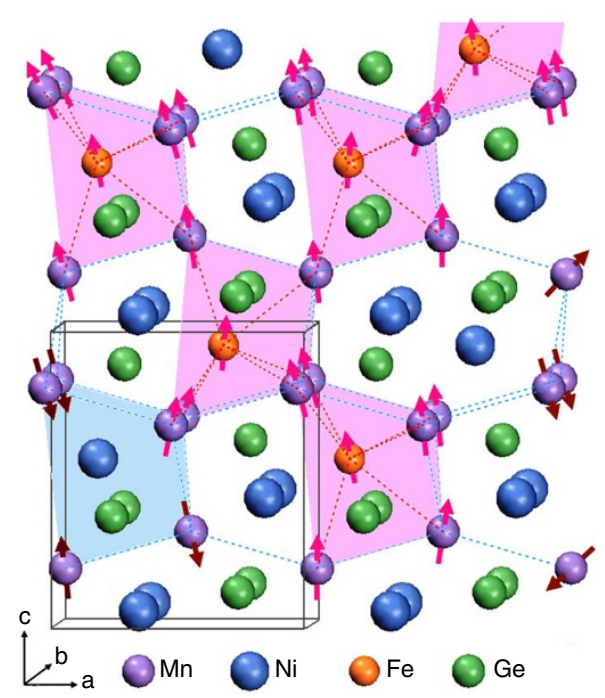

d

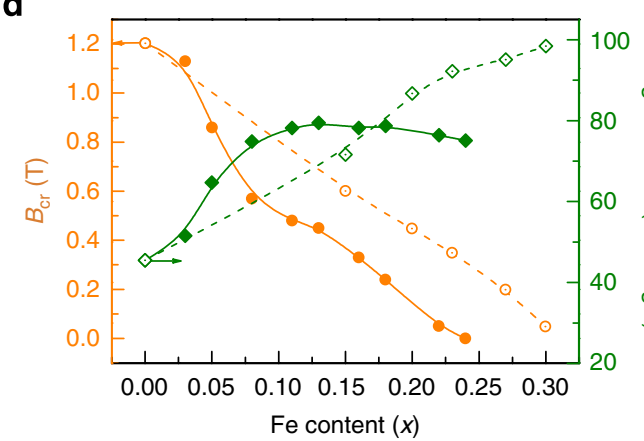

b

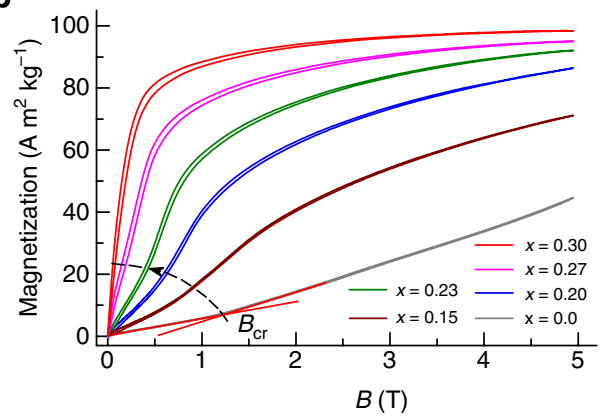

C

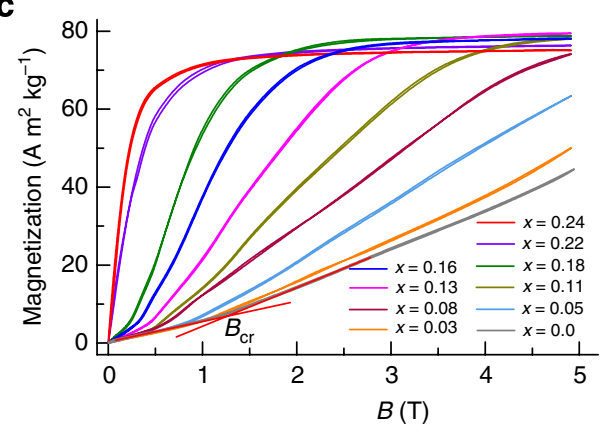

e

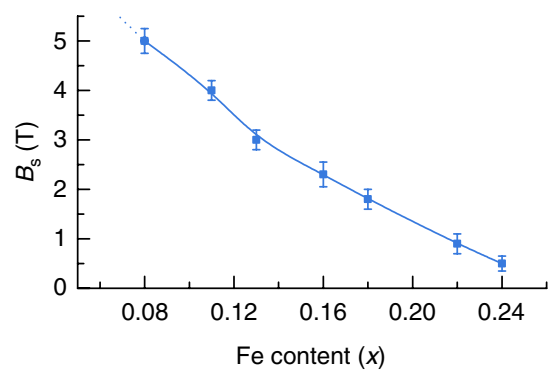

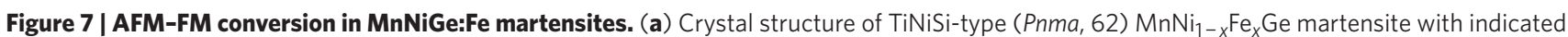
$\mathrm{Ni}-6 \mathrm{Mn}$ local configurations (light blue zones) and FM Fe-6Mn local configurations (pink zones). All atoms are at $4 \mathrm{c}(x, 1 / 4, z)$ positions 37,38 . The Fe atoms occupy $\mathrm{Ni}$ sites ${ }^{29}$ and each $\mathrm{Ni}(\mathrm{Fe})$ atom is surrounded by six nearest-neighbour $\mathrm{Mn}$ atoms. The arrows on $\mathrm{Mn}$ and Fe atoms illustrate the magnetic moments. (b,c) Experimental evidence of (spiral AFM)-FM conversion as a function of both Fe content $(x)$ and applied field $(B)$ for $\mathrm{MnNi}_{1-x} \mathrm{Fe}_{x} \mathrm{Ge}$ (b) and $\mathrm{MnNi}_{1-x} \mathrm{Fe}_{x} \mathrm{Ge}(\mathbf{c})$. The $M(B)$ curves in $(\mathbf{b}, \mathbf{c})$ have been measured in $5 \mathrm{~K}$ at which the samples are in the martensite state. The kink at $B_{\mathrm{cr}}$ in $(\mathbf{b}, \mathbf{c})$ corresponds to the metamagnetic critical field of the martensite. The dashed line in (b) indicates the decrease of $B_{\mathrm{cr}}$. (d) Dependence on the Fe-content of $B_{c r}$ (orange circles) and the magnetization in $5 \mathrm{~T}$ (green diamonds) of $\mathrm{MnNi}_{1-x} \mathrm{Fe}_{x} \mathrm{Ge}$ (open symbols) and $\mathrm{Mn}_{1-x} \mathrm{Fe}_{x} \mathrm{NiGe} \mathrm{solid} \mathrm{symbols}$ ) martensites. (e) Dependence on the Fe-content of the saturation field $\left(B_{\mathrm{S}}\right)$ of $\mathrm{Mn}_{1-\chi} \mathrm{Fe}_{\chi} \mathrm{NiGe}$ martensite. The error bars of the $B_{\mathrm{s}}$ are given by repeating the measurements.

This paper clearly clarifies the importance and tunability of magnetostructural coupling in these hexagonal ferromagnets.

To summarize, we have found that a stable PM-FM magnetostructural coupling in a broad temperature window, with tunable magnetoresponsive properties, can be obtained in martensitic phase-transition materials tailored by suitable substitution of $\mathrm{Fe}$ in $\mathrm{MnNiGe}$. It has been found that the MnNiGe can be manipulated in terms of crystallology and magnetism to be easily affected by an applied magnetic field. The MnNiGe:Fe material has been shown to possess compelling thermodynamic, crystallographic and magnetoresponsive effects with broad tunability in a broad temperature range. These effects may be utilized over potential smart applications working in the range between room temperature and liquid-nitrogen temperature, such as magnetic-field-controllable martensite particle/substrate composites ${ }^{3,48}$, solid-state magnetic refrigeration ${ }^{12,24,27}$ or multifunctional phase-transition-strain/ magnetic sensors ${ }^{15}$ jointly driven by both large-strain structural transition and sensitive magnetic switching from the PM to the FM state. The presented design scheme may be of importance in exploring multifunctional magnetoresponsive materials among new and known magnetic martensitic-transition systems.

\section{Methods}

Sample preparation. Polycrystalline ingots were prepared by arc melting highpurity metals in argon atmosphere. The ingots were melted four times and turned over in between to guarantee good alloying. The ingots were subsequently homogenized by annealing in evacuated quartz tubes under argon at $1,123 \mathrm{~K}$ for 5 days and slowly cooled at $1 \mathrm{~K} \mathrm{~min}^{-1}$ to room temperature to avoid stress in samples.

Structural and thermal analysis. The samples for powder XRD were made by fine grinding. The room-temperature powder XRD measurements were performed using a Rigaku XRD D/max 2,400 diffractometer with $\mathrm{Cu}-\mathrm{Ka}$ radiation. Temperature-dependent XRD measurements were performed from 285 to $98 \mathrm{~K}$ with a cooling rate of $2.5 \mathrm{~K} \mathrm{~min}^{-1}$ using a Bruker XRD D8-Advance diffractometer 
with $\mathrm{Cu}-\mathrm{Ka}$ radiation. At each temperature, a waiting time of $30 \mathrm{~min}$ was included before the measurement. DTA with heating and cooling rates of $2.5 \mathrm{~K} \mathrm{~min}^{-1}$ was used to determine the martensitic-transition characteristic temperatures.

Magnetic measurements. Magnetization measurements were carried out on powder samples using a superconducting quantum interference device (SQUID, Quantum Design MPMS XL-7). Low-field $M(T)$ measurements were performed to study the temperature-dependent magnetic behaviour of samples and also to determine the martensitic-transition characteristic temperatures. These experiments were combined with DTA. ZFC-FC thermomagnetization in a field of $0.01 \mathrm{~T}$ and frequency-dependent magnetic susceptibility were measured with frequencies $f=1$, $97,496,997$ and $1,488 \mathrm{~Hz}$ in an AC magnetic field of 4 Oe after ZFC from $300 \mathrm{~K}$.

To accurately derive the magnetic-entropy changes $\left(\Delta S_{\mathrm{m}}\right)$ at magnetostructural transitions with thermal hysteresis, the so-called loop process method ${ }^{49}$ was adopted to get the isothermal magnetization curves. The isothermal $M(B)$ curves were measured in fields of up to $5 \mathrm{~T}$ upon cooling with a temperature interval of $2 \mathrm{~K}$. Before each isothermal magnetization, the samples were all the way heated up to the complete PM austenite region $(100 \mathrm{~K}$ away from the magnetostructural transitions) with heating rate of $5 \mathrm{~K} \mathrm{~min}^{-1}$ to eliminate the history-dependent magnetic states and then cooled back to the targeted measurement temperature points. All these temperature loops were performed in zero field. To avoid the overmuch temperature-induced FM martensite phase during approaching each targeted temperature point, the cooling rate was set as $2 \mathrm{~K} \mathrm{~min}^{-1}$. Besides, a waiting time of $300 \mathrm{~s}$ was compelled before the measurements to guarantee a highly stable temperature. The $\Delta S_{\mathrm{m}}$ was then derived from the resulting magnetization curves using the Maxwell relation ${ }^{50}$

$$
\Delta S_{\mathrm{m}}(T, H)=S_{\mathrm{m}}(T, H)-S_{\mathrm{m}}(T, 0)=\int_{0}^{H}\left(\frac{\partial M}{\partial T}\right)_{\mathrm{H}} \mathrm{d} H
$$

ELF calculations. ELF calculations were carried out using the pseudopotential method with plane-wave-basis set based on the density-functional theory ${ }^{51}$. The electronic exchange correlation energy was treated under the local spin density approximation ${ }^{52}$. Plane-wave cutoff energy of $770 \mathrm{eV}$ and $126(13 \times 13 \times 12) \mathrm{k}$ points in the irreducible Brillouin zone were used for a good convergence of the total energy. The absolute total-energy difference tolerance for the self-consistent field cycle was set at $5 \times 10^{-7} \mathrm{eV} /$ atom. The geometry optimizations for the atomic site occupancy in the cell were performed on the experimental lattice parameters using the Broyden-Fletcher-Goldfarb-Shanno minimization scheme ${ }^{53}$. The ELF ${ }^{43}$, as an indicator of the electron-pair distribution in terms of inter-atomic bonding, is introduced to represent the conditional probability of finding a second like-spin electron near the reference position. It is a local, relative measure of the Pauli repulsion effect on the kinetic energy density. The topological analysis of ELF represents the organization of chemical bonds and, more particularly, the bond types. Higher ELF values at the reference position show that the electrons are more localized than in a uniform electron gas of identical density, which indicates a stronger covalent bonding between neighbouring atoms in alloys and compounds.

\section{References}

1. Kokorin, V. V. \& Chernenko, V. A. The martensitic transformation in a ferromangetic Heusler alloy. Phys. Met. Metall. 68, 111-115 (1989).

2. Tanaka, Y. et al. Ferrous polycrystalline shape-memory alloy showing huge superelasticity. Science 327, 1488-1490 (2010).

3. Liu, J., Scheerbaum, N., Weiss, S. \& Gutfleisch, O. Ni-Mn-In-Co singlecrystalline particles for magnetic shape memory composites. Appl. Phys. Lett. 95, 152503 (2009)

4. Ullakko, K., Huang, J. K., Kantner, C., O’Handley, R. C. \& Kokorin, V. V. Large magnetic-field-induced strains in $\mathrm{Ni}_{2} \mathrm{MnGa}$ single crystals. Appl. Phys. Lett. 69, 1966-1968 (1996)

5. Wu, G. H. et al. Giant magnetic-field-induced strains in Heusler alloy NiMnGa with modified composition. Appl. Phys. Lett. 75, 2990-2992 (1999).

6. Kakeshita, T. et al. Magnetic field-induced martensitic transformation and giant magnetostriction in Fe-Ni-Co-Ti and ordered $\mathrm{Fe}_{3} \mathrm{Pt}$ shape memory alloys. Mater. T. Jim. 41, 882-887 (2000).

7. Kainuma, R. et al. Magnetic-field-induced shape recovery by reverse phase transformation. Nature 439, 957-960 (2006).

8. Yu, S. Y. et al. Large magnetoresistance in single-crystalline $\mathrm{Ni}_{50} \mathrm{Mn}_{50-x} \mathrm{In}_{x}$ alloys $(x=14-16)$ upon martensitic transformation. Appl. Phys. Lett. 89, 162503 (2006).

9. Barandiaran, J. M., Chernenko, V. A., Lazpita, P., Gutierrez, J. \& Feuchtwanger, J. Effect of martensitic transformation and magnetic field on transport properties of Ni-Mn-Ga and Ni-Fe-Ga Heusler alloys. Phys. Rev. B 80, 104404 (2009).

10. Dubenko, I. et al. Giant Hall effect in Ni-Mn-In Heusler alloys. Phys. Rev. B 80 092408 (2009).

11. Krenke, T. et al. Inverse magnetocaloric effect in ferromagnetic Ni-Mn-Sn alloys. Nat. Mater. 4, 450-454 (2005).

12. Gutfleisch, O. et al. Magnetic materials and devices for the 21st century: stronger, lighter, and more energy efficient. Adv. Mater. 23, 821-842 (2011).
13. Karaca, H. E. et al. Magnetic field-induced phase transformation in NiMnCoIn magnetic shape-memory alloys-a new actuation mechanism with large work output. Adv. Funct. Mater. 19, 983-998 (2009).

14. Chmielus, M., Zhang, X. X., Witherspoon, C., Dunand, D. C. \& Mullner, P. Giant magnetic-field-induced strains in polycrystalline Ni-Mn-Ga foams. Nat. Mater. 8, 863-866 (2009).

15. Sarawate, N. \& Dapino, M. Experimental characterization of the sensor effect in ferromagnetic shape memory Ni-Mn-Ga. Appl. Phys. Lett. 88, 121923 (2006).

16. Karaman, I., Basaran, B., Karaca, H. E., Karsilayan, A. I. \& Chumlyakov, Y. I. Energy harvesting using martensite variant reorientation mechanism in a NiMnGa magnetic shape memory alloy. Appl. Phys. Lett. 90, 172505 (2007).

17. Manosa, L. et al. Giant solid-state barocaloric effect in the Ni-Mn-In magnetic shape-memory alloy. Nat. Mater. 9, 478-481 (2010).

18. Dung, N. H., Zhang, L., Ou, Z. Q. \& Brück, E. From first-order magneto-elastic to magneto-structural transition in $(\mathrm{Mn}, \mathrm{Fe})_{1.95} \mathrm{P}_{0.50} \mathrm{Si}_{0.50}$ compounds. Appl. Phys. Lett. 99, 092511 (2011).

19. Pecharsky, V. K. \& Gschneidner, J. K. A. Giant magnetocaloric effect in $\mathrm{Gd}_{5}\left(\mathrm{Si}_{2} \mathrm{Ge}_{2}\right)$. Phys. Rev. Lett. 78, 4494-4497 (1997).

20. Pecharsky, V. K. \& Gschneidner, K. A. $\mathrm{Gd}_{5}\left(\mathrm{Si}_{x} \mathrm{Ge}_{1-x}\right)_{4}$ : an extremum material. Adv. Mater. 13, 683-686 (2001).

21. Pecharsky, V. K., Holm, A. P., Gschneidner, K. A. \& Rink, R. Massive magneticfield-induced structural transformation in $\mathrm{Gd}_{5} \mathrm{Ge}_{4}$ and the nature of the giant magnetocaloric effect. Phys. Rev. Lett. 91, 197204 (2003).

22. Provenzano, V., Shapiro, A. J. \& Shull, R. D. Reduction of hysteresis losses in the magnetic refrigerant $\mathrm{Gd}_{5} \mathrm{Ge}_{2} \mathrm{Si}_{2}$ by the addition of iron. Nature 429, 853-857 (2004)

23. Wada, H. \& Tanabe, Y. Giant magnetocaloric effect of $\mathrm{MnAs}_{1-x} \mathrm{Sb}_{x}$. Appl. Phys. Lett. 79, 3302-3304 (2001)

24. de Campos, A. et al. Ambient pressure colossal magnetocaloric effect tuned by composition in $\mathrm{Mn}_{1-}{ }_{x} \mathrm{Fe}_{x}$ As. Nat. Mater. 5, 802-804 (2006).

25. Hu, F. X. et al. Influence of negative lattice expansion and metamagnetic transition on magnetic entropy change in the compound $\mathrm{LaFe}_{11.4} \mathrm{Si}_{1.6}$. Appl. Phys. Lett. 78, 3675-3677 (2001).

26. Fujieda, S., Fujita, A. \& Fukamichi, K. Large magnetocaloric effects in $\mathrm{NaZn}_{13}$ type $\mathrm{La}\left(\mathrm{Fe}_{x} \mathrm{Si}_{1-x}\right)_{13}$ compounds and their hydrides composed of icosahedral clusters. Sci. Technol. Adv. Mater. 4, 339-346 (2003).

27. Tegus, O., Brück, E., Buschow, K. H. J. \& de Boer, F. R. Transition-metal-based magnetic refrigerants for room-temperature applications. Nature 415, 150-152 (2002).

28. Koyama, K., Sakai, M., Kanomata, T. \& Watanabe, K. Field-induced martensitic transformation in new ferromagnetic shape memory compound $\mathrm{Mn}_{1.07} \mathrm{Co}_{0.92}$ Ge. Jpn. J. Appl. Phys. Part 1 43, 8036-8039 (2004).

29. Szytula, A., Pedziwiatr, A. T., Tomkowicz, Z. \& Bażeła, W. Crystal and magnetic structure of CoMnGe, CoFeGe, FeMnGe and NiFeGe. J. Magn. Magn. Mater. 25, 176-186 (1981)

30. Tremel, W., Hoffmann, R. \& Silvestre, J. Transitions between NiAs and MnP type phases - an electronically driven distortion of triangular $\left(3^{6}\right)$ nets. J. Am. Chem. SOC. 108, 5174-5187 (1986).

31. Zhang, H. J. et al. Topological insulators in ternary compounds with a honeycomb lattice. Phys. Rev. Lett. 106, 156402 (2011).

32. Zhang, C. L. et al. Magnetostructural phase transition and magnetocaloric effect in off-stoichiometric $\mathrm{Mn}_{1.9-} \mathrm{Ni}_{x} \mathrm{Ge}$ alloys. Appl. Phys. Lett. 93, 122505 (2008).

33. Liu, E. K. et al. Vacancy-tuned paramagnetic/ferromagnetic martensitic transformation in Mn-poor $\mathrm{Mn}_{1-x}$ CoGe alloys. EPL 91, 17003 (2010).

34. Trung, N. T., Zhang, L., Caron, L., Buschow, K. H. J. \& Brück, E. Giant magnetocaloric effects by tailoring the phase transitions. Appl. Phys. Lett. 96, 172504 (2010).

35. Trung, N. T. et al. From single- to double-first-order magnetic phase transition in magnetocaloric $\mathrm{Mn}_{1-x} \mathrm{Cr}_{x}$ CoGe compounds. Appl. Phys. Lett. 96, 162507 (2010).

36. Liu, E. K. et al. Magnetostructural transformation and magnetoresponsive properties of $\mathrm{MnNiGe}_{1-x} \mathrm{Sn}_{x}$ alloys. IEEE Trans. Magn. 47, 4041-4043 (2011)

37. Bażela, W., Szytuła, A., Todorovć, J., Tomkowicz, Z. \& Zięba, A. Crystal and magnetic structure of NiMnGe. Phys. Status Solidi A 38, 721-729 (1976).

38. Fjellvåg, H. \& Andresen, A. F. On the crystal structure and magnetic properties of MnNiGe. J. Magn. Magn. Mater. 50, 291-297 (1985).

39. Johnson, V. Diffusionless orthorhombic to hexagonal transitions in ternary silicides and germanides. Inorg. Chem. 14, 1117-1120 (1975).

40. Cui, J. et al. Combinatorial search of thermoelastic shape-memory alloys with extremely small hysteresis width. Nat. Mater. 5, 286-290 (2006).

41. Brück, E. Developments in magnetocaloric refrigeration. J. Phys. D Appl. Phys. 38, R381-R391 (2005).

42. Planes, A., Mañosa, L. \& Acet, M. Magnetocaloric effect and its relation to shape-memory properties in ferromagnetic Heusler alloys. J. Phys. Condens. Matter 21, 233201 (2009)

43. Becke, A. D. \& Edgecombe, K. E. A simple measure of electron localization in atomic and molecular systems. J. Chem. Phys. 92, 5397-5403 (1990).

44. Sredniawa, B. et al. Crystal structure, magnetic and electronic properties of $\mathrm{Co}_{x} \mathrm{Fe}_{1-x} \mathrm{MnP}$ system. J. Alloy. Compd. 317-318, 266-273 (2001). 
45. Nizioł, S., Bombik, A., Bażela, W., Szytuła, A. \& Fruchart, D. Crystal and magnetic structure of $\mathrm{Co}_{x} \mathrm{Ni}_{1-x} \mathrm{MnGe}$ system. J. Magn. Magn. Mater. 27, 281-292 (1982).

46. Bażela, W., Szytuła, A., Todorovć, J. \& Zięba, A. Crystal and magnetic structure of the $\mathrm{NiMnGe}_{1-n} \mathrm{Si}_{n}$ system. Phys. Status Solidi A 64, 367-378 (1981).

47. Caron, L., Trung, N. T. \& Brück, E. Pressure-tuned magnetocaloric effect in $\mathrm{Mn}_{0.93} \mathrm{Cr}_{0.07}$ CoGe. Phys. Rev. B 84, 020414 (2011).

48. Kirkby, E. L. et al. Embedded shape-memory alloy wires for improved performance of self-healing polymers. Adv. Funct. Mater. 18, 2253-2260 (2008).

49. Caron, L. et al. On the determination of the magnetic entropy change in materials with first-order transitions. J. Magn. Magn. Mater. 321, 3559-3566 (2009).

50. Foldeaki, M., Chahine, R. \& Bose, T. K. Magnetic measurements: a powerful tool in magnetic refrigerator design. J. Appl. Phys. 77, 3528-3537 (1995).

51. Payne, M. C., Teter, M. P., Allan, D. C., Arias, T. A. \& Joannopoulos, J. D. Iterative minimization techniques for $a b$ initio total-energy calculations: molecular dynamics and conjugate gradients. Rev. Mod. Phys. 64, 1045-1097 (1992).

52. Perdew, J. P. \& Zunger, A. Self-interaction correction to density-functional approximations for many-electron systems. Phys. Rev. B 23, 5048-5079 (1981).

53. Fischer, T. H. \& Almlof, J. General methods for geometry and wave function optimization. J. Phys. Chem. 96, 9768-9774 (1992).

\section{Acknowledgements}

We gratefully acknowledge Dr. Yu Wang (Xian Jiaotong University, China) for kind help in temperature-dependent XRD measurements, Prof. Xiaobing Ren (NIMS, Japan) and
Prof. V. A. Chernenko (Universidad del País Vasco, Spain) for fruitful discussions. This work was supported by the National Natural Science Foundation of China (51031004, 51021061 and 11174352) and National Basic Research Program of China (973 Programs: 2009CB929501, 2010CB833102 and 2012CB619405).

\section{Author contributions}

Experiments were conceived by E.K.L. and W.H.W. and carried out by E.K.L., L.F., W.Z. and G.J.L. with assistance of J.L.C. Results were analysed and interpreted by E.K.L., W.H.W., L.F., W.Z., G.J.L., G.H.W., H.W.Z. and F.R.deB. ELF calculations were performed by E.K.L., C.B.J. and H.B.X. The manuscript was written by E.K.L., G.H.W., W.H.W. and F.R.deB. W.H.W., H.W.Z. and G.H.W. are responsible for project direction, planning and infrastructure.

\section{Additional information}

Supplementary Information accompanies this paper at http://www.nature.com/ naturecommunications

Competing financial interests: The authors declare no competing financial interests.

Reprints and permission information is available online at http://npg.nature.com/ reprintsandpermissions/

How to cite this article: Liu, E. et al. Stable magnetostructural coupling with tunable magnetoresponsive effects in hexagonal ferromagnets. Nat. Commun. 3:873 doi: 10.1038/ncomms1868 (2012).

License: This work is licensed under a Creative Commons Attribution-NonCommercialShare Alike 3.0 Unported License. To view a copy of this license, visit http:// creativecommons.org/licenses/by-nc-sa/3.0/ 